\title{
Banco de pruebas experimental para esquemas de control por cambio del ángulo de paso en aerogeneradores
}

\author{
Experimental testing bench for variable \\ pitch wind turbines control strategies \\ Miguel Á. Hernández López ${ }^{1}$ Rosendo Rosales López ${ }^{1}$ \\ J. Jesús Arellano Pimentel ${ }^{2 *} \quad$ Francisco Aguilar Acevedo ${ }^{2} \quad$ Aline Ramírez Jaramillo $^{3}$ \\ Recibido 28 de agosto de 2018, aceptado 30 de octubre de 2019 \\ Received: August 28, 2018 Accepted: October 30, 2019
}

\begin{abstract}
RESUMEN
El crecimiento a nivel mundial de la demanda energética exige el diseño y construcción de sistemas de generación cada vez más eficientes. En esta labor los esquemas de control juegan un papel preponderante y contar con un sistema que permita evaluar de forma experimental su desempeño se vuelve clave. En el caso de la energía eólica uno de los medios de control es la variación del ángulo de ataque de las palas de los aerogeneradores. En este trabajo, se presentan los resultados del diseño y construcción de un banco de pruebas para llevar a cabo la evaluación experimental de esquemas de control por cambio del ángulo en aerogeneradores de baja potencia. Mediante pruebas en un túnel de viento se caracterizó la operación del rotor y el de cambio del ángulo de paso. La velocidad del viento para que el rotor comience a operar fue de $3,5 \mathrm{~m} / \mathrm{s}$. Se implementaron y probaron dos controladores, uno PI y uno difuso.
\end{abstract}

Palabras clave: Banco de pruebas, aerogeneradores, cambio del ángulo de paso, rotor, algoritmos de control.

\begin{abstract}
The growing worldwide energy demand requires the design and construction of increasingly efficient generation systems. In satisfying this need, control schemes play an essential role. Having a system that allows experimental evaluation of their performance is key. In the case of wind energy, one possible control strategy is pitch regulation, which is used in variable pitch wind turbine systems. This paper presents the results of the design and construction of a testing bench for carrying out the experimental evaluation of control strategies for pitch regulated small wind turbines. Rotor operation and pitch regulation were ascertained through wind tunnel testing. Rotors start moving with winds of $3.5 \mathrm{~m} / \mathrm{s}$. PI and fuzzy controllers were implemented and tested.
\end{abstract}

Keywords: Testing bench, wind turbines, variable pitch, rotor, control algorithms.

\footnotetext{
1 Universidad del Istmo. Instituto de Estudios de la Energía. Sto. Dom. Tehuantepec, Oaxaca, México. E-mail: mahluni2000@hotmail.com; rosendo.rosales1992@hotmail.com

2 Universidad del Istmo. Ingeniería en Computación. Sto. Dom. Tehuantepec, Oaxaca, México.

E-mail: jjap@sandunga.unistmo.edu.mx; aguilar.afco@sangunga.unistmo.edu.mx

3 Team Builder S.R.L. México. E-mail: aline.ramij@gmail.com

* Autor de correspondencia: jjap@sandunga.unistmo.edu.mx
} 


\section{INTRODUCCIÓN}

En los últimos años el sector eólico se ha desarrollado de forma acelerada, abriendo con ello nuevas líneas de investigación o creando nuevos paradigmas. Lo anterior conlleva dos propósitos principales: el primero, aumentar la energía producida y mejorar la calidad de la señal generada; el segundo, es incrementar la eficiencia y alargar la vida útil de las máquinas y equipos utilizados.

Actualmente, el principal sistema empleado para llevar a cabo el aprovechamiento de la energía cinética del viento son los aerogeneradores. Estos se pueden clasificar en dos grupos primordiales: los de eje vertical (VAWT) y los de eje horizontal (HAWT). Siendo los segundos, en su configuración de tres palas, los mayormente empleados en el proceso de generación de energía eléctrica. Las principales topologías de aerogeneradores de eje horizontal son dos y se definen por el tipo de generador eléctrico que emplean. Por un lado, están los que integran generadores síncronos tipo multipolo y por otro los que emplean generadores de inducción doblemente alimentados con multiplicadora de velocidad. Dentro de ambas topologías, se pueden encontrar elementos esenciales (Figura 1) agrupados en subsistemas mecánico, eléctrico, control, monitoreo, y estructural.

Los esquemas de control para los diferentes subsistemas son fundamentales en el incremento de la eficiencia en aerogeneradores. En el sector eólico industrial un aerogenerador presenta tres etapas de control de acuerdo con su curva de potencia [1]. La primera, referente a la respuesta transitoria hasta alcanzar la velocidad nominal. La segunda mantiene el aerogenerador dentro de la velocidad nominal y la tercera se presenta cuando la velocidad del

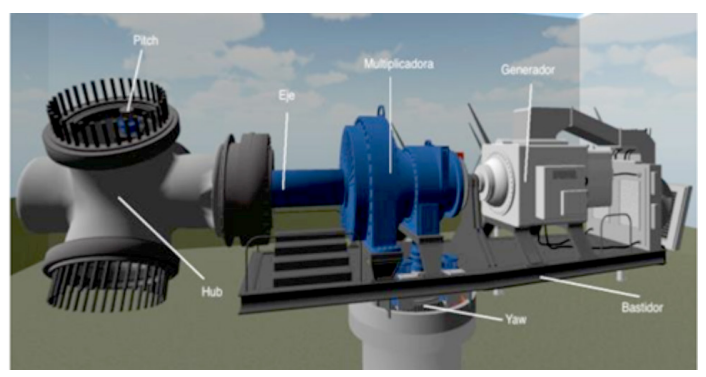

Figura 1. Aerogenerador y sus componentes principales. viento es superior a la velocidad de corte y debe de interrumpirse la operación del aerogenerador.

En la segunda etapa de operación el control del sistema se mantiene por medio de la regulación del ángulo de ataque de las palas del rotor (ángulo de paso). Con la variación del ángulo se busca mantener la velocidad de giro del rotor constante y absorber las variaciones en la velocidad del viento mientras no se alcance la velocidad de desconexión. Durante la operación a velocidad nominal el sistema de cambio del ángulo es el principal encargado de controlar y regular la potencia producida.

Diferentes esquemas de control por cambio del ángulo han sido propuestos desde hace varios años atrás $[2,3,4,5,6,7]$, aunque en la mayoría de los casos sólo son evaluados por medio de simulación matemática; por ejemplo, en [2] se presenta un esquema de control por cambio del ángulo aplicando una red neuronal de base radial y empleado como método de diseño el análisis de estabilidad de Lyapunov, validando sus resultados a través de un simulador de un aerogenerador de $5 \mathrm{MW}$. Por su parte, en [3] simulan la propuesta del diseño de un sistema de cambio del ángulo de paso basado en un esquema de control por modelo interno de dos grados de libertad utilizando la herramienta Matlab/ Simulink. Empleando el citado software, en [4] se simula un controlador PID para un sistema de control de pitch variable con actuadores hidráulicos, mientras en [5] analizan el desempeño de un controlador PI y otro basado en lógica difusa para el control por cambio del ángulo en un aerogenerador con generador síncrono de imán permanente, y muestran simulaciones con las que concluyen que el torque de referencia es convenientemente logrado ajustando el ángulo de pitch, independientemente del controlador. De manera similar, en [6] analizan los resultados de la simulación de dos controladores: PI y redes neuronales; alcanzando resultados similares a los presentados en [5]. Novedosos esquemas de control de potencia que implican la regulación del pitch también han sido abordados a través de la simulación como en [7].

Recientemente algunos trabajos han validado esquemas de control para cambio del ángulo. Por ejemplo, en [8] se diseña y evalúa un sistema de pitch tipo bisagra (hinge) para pequeños aerogeneradores. El banco de prueba consta de una estructura hinge 
donde se empotran tres palas, un generador, y un actuador a través del cual se realiza el cambio del ángulo de paso. Por su parte, en [9], se emplea un simulador de $3,5 \mathrm{~kW}$ para emular un controlador pitch de un aerogenerador de $2 \mathrm{MW}$. El simulador consta de un motor, una caja de engranes, un volante de inercia (flywheel) y un generador. El torque aerodinámico es implementado a través del motor. Bajo el mismo esquema, en $[10,11]$ se emplea un motor, un volante de inercia, y un generador, para estudiar respectivamente, los efectos de la emulación de aerogeneradores con alta inercia, y poner a prueba una nueva estrategia de control de potencia activa que integra la regulación de la velocidad del rotor y del ángulo de pitch. Mientras, en [12] se estudian los efectos del pitch en el rendimiento de pequeños aerogeneradores en zonas urbanas, mediante un prototipo montado en una torre de $5 \mathrm{~m}$ de altura, que consiste en un rotor de tres palas acoplado a un eje, al que es conectado un transductor de par, y un interruptor de seguridad (freno mecánico), no obstante, el posicionamiento ángulo de paso se realiza de manera manual. En el mismo sentido, en [13] se emplea un modelo a escala de 1:100 para un aerogenerador de eje horizontal offshore de $5 \mathrm{MW}$, comparan los resultados de dos controladores de cambio del ángulo, uno PI y otro $\mathrm{H} \infty$. Al tratarse de un aerogenerador offshore su objetivo es reducir el movimiento inducido por el viento sobre la plataforma flotante. Por su lado, en [14] se investigan los efectos de un ajuste de pitch dinámico en aerogeneradores a pequeña escala de tipo vertical. No obstante, también analizan los efectos de diferentes ángulos de pitch para aerogeneradores de eje horizontal, para tales fines utilizan una impresora 3D con el propósito de generar un conjunto de hub's que permiten empotrar palas en diferentes ángulos de ataque. En [15] también realizan un estudio sobre los parámetros de salida de un HAWT producidas por la variación del ángulo de pitch y variación del largo, ancho, peso y modelo aerodinámico de las palas. Para variar el ángulo de pitch proponen un diseño mecánico en el hub, el cual puede ser ajustado manualmente desde $0^{\circ}$ hasta $180^{\circ}$. Dentro de estos estudios, otra alternativa explorada es la emulación mediante hardware, como como plataformas CPU/GPU [16]. Los sistemas de pitch también son usados en turbinas de corriente marina (tidal current turbines), como en [17] donde se aborda su estudio a través de un banco de pruebas con actuadores hidráulicos.
Si bien, la mayoría de los esquemas de control diseñados se evalúan primero en simulación por diferentes métodos, la implementación física de los mismos siempre presentará retos a resolver. Poder diseñar esquemas de control del sistema de cambio del ángulo de paso y evaluar su desempeño de manera experimental se torna complicado si se consideran los altos costos que tendría adquirir la infraestructura necesaria. A continuación, se presenta una propuesta de banco experimental de pruebas para la evaluación del desempeño de sistemas de control por variación de ángulo de ataque. De manera inherente el banco permitirá valorar la operación de diferentes juegos de palas.

\section{DISEÑO Y CONSTRUCCIÓN DEL BANCO EXPERIMENTAL DE PRUEBAS}

Siguiendo la propuesta metodológica iterativa de [18] se establecieron cuatro etapas en el desarrollo del banco experimental. En la primera, se definieron los requerimientos y restricciones, dentro de las cuales destaca el número de palas, establecido como tres (tri-pala), al ser el de mayor empleo en aerogeneradores de alta potencia. En la segunda, se desarrolló un diseño conceptual para establecer las estructuras funcionales y primeras propuestas de solución. Posteriormente se procedió a la tercera etapa, que correspondió al diseño de forma para establecer el producto con las consideraciones técnicas y económicas específicas. Finalmente se procedió a la fase del diseño de detalle, en donde se determinaron los componentes finales, sus dimensiones y materiales. Una vez terminando este punto, se volvió a verificar el cumplimiento de los aspectos técnicos, económicos y se analizó la factibilidad del diseño presentado, para proceder a la presentación de planos y documentos para la fabricación de piezas en los casos pertinentes.

El banco experimental está constituido por dos subsistemas: mecánico, y control-monitoreo (Figura 2). El subsistema mecánico está compuesto por un eje (de baja velocidad) soportado a la base con dos rodamientos tipo chumacera, al eje se ensambla el cubo o hub al que se acopla el juego de tres palas. Como aerogenerador este banco puede ser clasificado como una microturbina, por lo que en este diseño no se considera la existencia de una multiplicadora. 


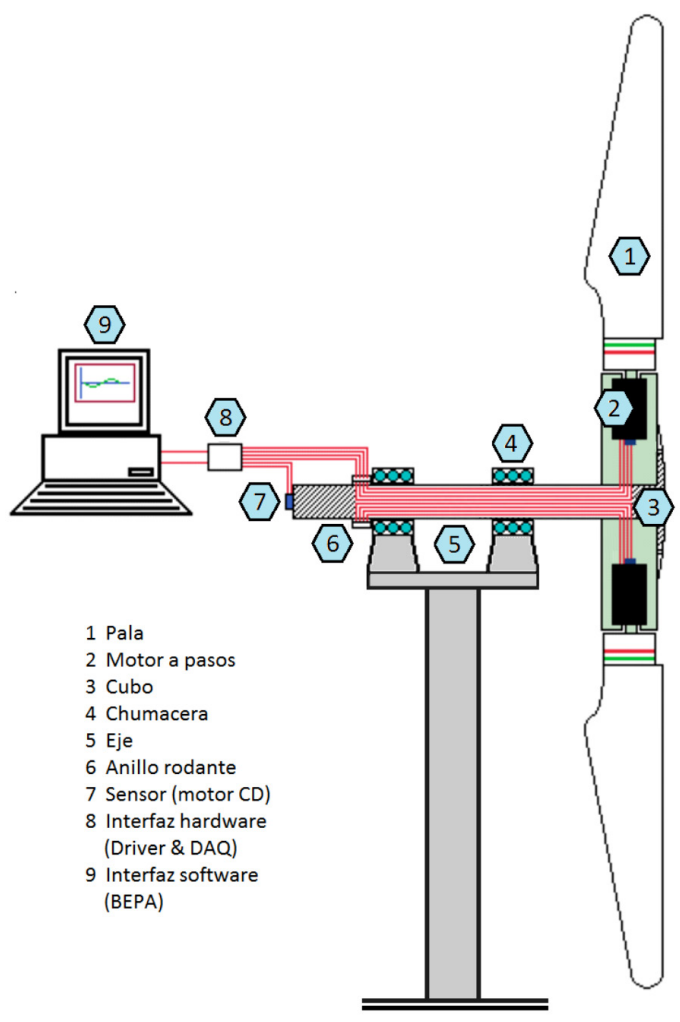

Figura 2. Componentes del banco experimental de pruebas.

En lo correspondiente al subsistema de controlmonitoreo se consideran los mecanismos de pitch de las palas, los actuadores eléctricos (motores a pasos con resolución de $1,8^{\circ}$ por paso), los sensores ópticos para el posicionamiento inicial de los motores a pasos, los sensores para la medición de la velocidad de giro del rotor y viento, y la integración de un sistema SCADA llamado BEPA [19] que permiten entre otras funciones conocer el estado de operación del banco y modificar el ángulo de pitch para cada pala. La estructura de soporte del banco está construida en acero y cuenta con elementos móviles en su parte inferior para facilitar su traslado.

La medición de la velocidad de rotación del eje en una primera iteración, se planteó mediante el uso de un enconder Applied Motion, no obstante en busca de una simplificación técnica y económica del diseño, se optó en una segunda iteración por utilizar un motor de corriente directa acoplado al eje en la parte posterior, permitiendo un cálculo indirecto de la velocidad de rotación del rotor.

El diseñó del cubo permitió el acoplamiento con el eje de giro, y el alojamiento de los tres motores y sensores ópticos para la posición de cada pala (Figura 3). La fabricación del cubo se realizó en nylamid mediante un proceso de maquinado CNC. $\mathrm{La}$ selección del material obedece a sus características de ligereza, resistencia a la corrosión, y facilidad de mecanizado.

Para suministrar energía eléctrica y operar los motores se realizaron modificaciones en el eje original, incorporando dos canales a los costados a través de los cuales se hacen pasar los cables de alimentación y control. Además, en la parte posterior del eje de $812,8 \mathrm{~mm}$ (32 pulgadas) de longitud se colocó un anillo rodante de 24 hilos de la marca MOOG, que evita que los cables se enreden (Figura 4).

En el manejo de los motores a pasos NEMA 34 HT34-506 de 200 pasos/rev y $8 \mathrm{Nm}$ de torque, que son utilizados para el cambio del pitch se hizo uso del driver ST5 del mismo fabricante de los motores

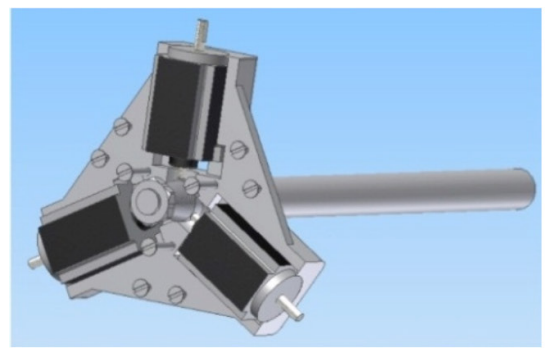

(a)

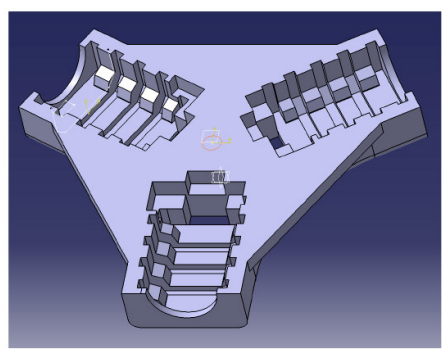

(b)

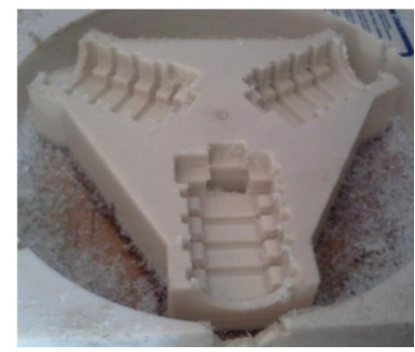

(c)

Figura 3. Diseño y fabricación del cubo para contener los actuadores para el cambio del pitch. (a) Ensamble de motores con cubo en CAD, (b) CAD del cubo, (c) cubo fabricado. 


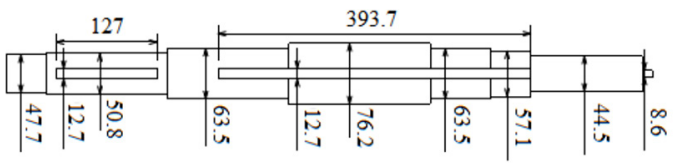

(a)

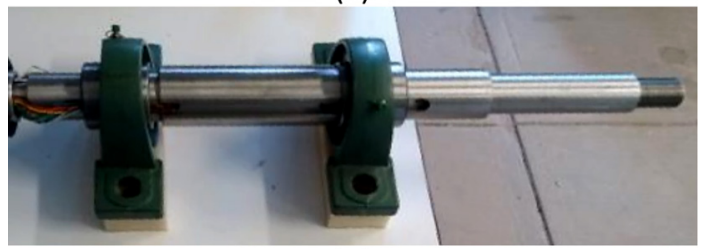

(b)

Figura 4. Diseño e implementación del eje (Unidades: milímetros). (a) plano del eje, (b) eje fabricado.

(Applied Motion). Cabe señalar que la combinación de motores a pasos y drives seleccionados permitió simplificarla operación de motores, que por sí mismos presentan como ventajas una alta precisión y repetibilidad. Los sensores ópticos en las palas permiten posicionar las palas en un punto de referencia para el movimiento relativo de los motores a pasos, permitiendo así establecer un movimiento absoluto para el ángulo de ataque para cada pala.

La opción tecnológica seleccionada para realizar la adquisición de datos fue un sistema NI cDAQ 9172 con las tarjetas, NI 9205 y NI9401 de la firma National Instrument (Figura 5). El costo y la asociación directa de este hardware con el software de instrumentación Labview bajo el cual fue desarrollado sistema BEPA [19], hacen a está una opción viable.

\section{PRUEBAS FUNCIONALES DEL BANCO EXPERIMENTAL}

Con el objeto de evaluar la respuesta del sistema en lazo abierto y los efectos de la regulación del ángulo de paso se realizaron dos tipos de pruebas. En ambas se empleó un túnel de viento del Instituto Nacional de Electricidad y Energías Limpias (INEEL) que permite registrar velocidades de 1,2 a $10 \mathrm{~m} / \mathrm{s}$. El banco opera en modo sotavento (Figura 6), la cual es una configuración muy recurrida en aerogeneradores de baja potencia. La operación del aerogenerador en un ambiente no controlado registró movimiento del rotor a velocidades inferiores a los 4,5 m/s (Figura 7).

\section{Caracterización de la operación del rotor}

De manera intencionada el conjunto de palas utilizadas carece de un perfil aerodinámico identificado, por lo cual fue necesario localizar experimentalmente el ángulo óptimo que permitiera el máximo aprovechamiento del recurso eólico, el procedimiento consistió en variar gradualmente el ángulo de paso bajo condiciones de velocidad constante del viento, buscando el punto de operación donde la velocidad del eje fuera máxima. Para el

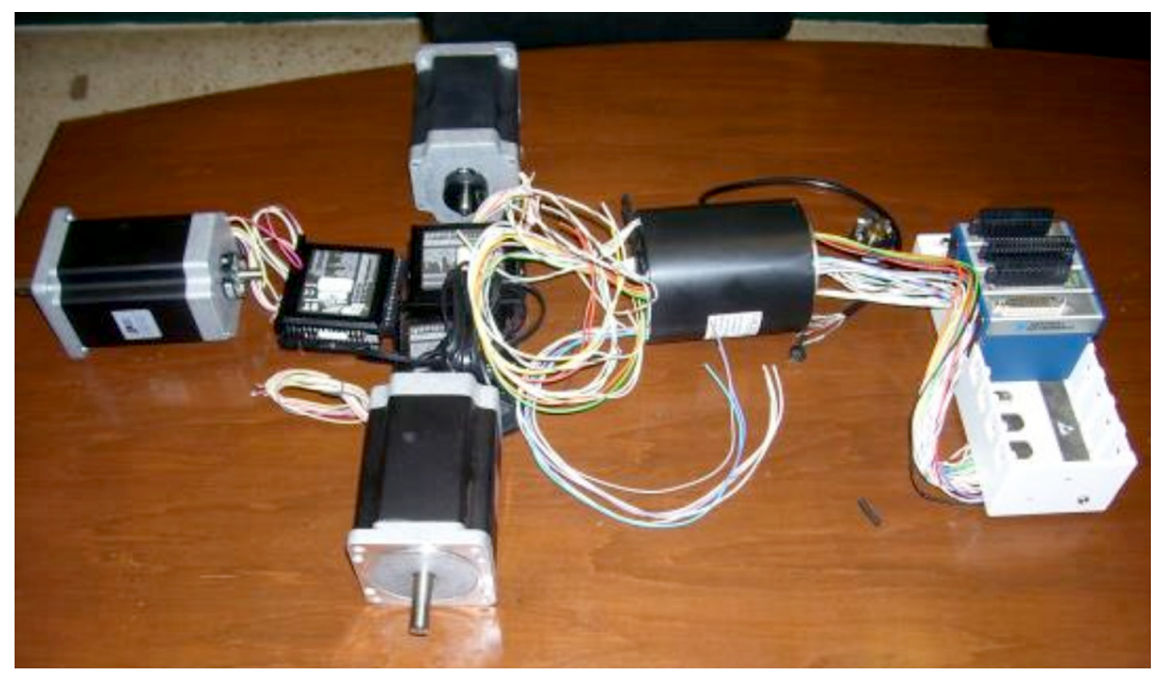

Figura 5. Sistema NI cDAQ 9172 con las tarjetas NI 9205 y NI 9401 con motores a pasos empleados en el cambio del pitch. 


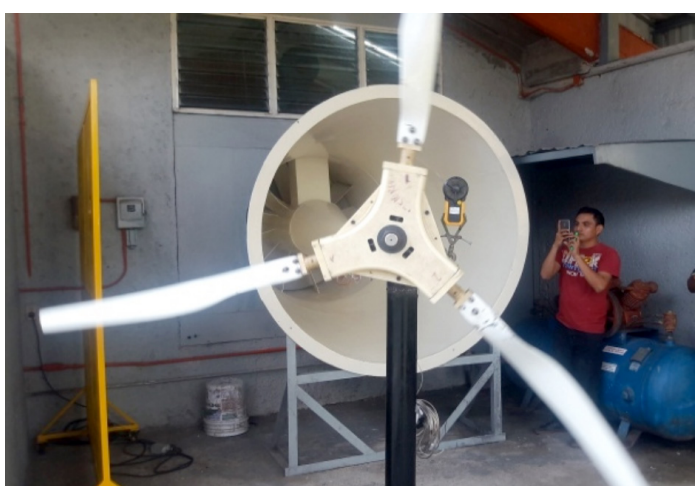

Figura 6. Operación del banco en un túnel de viento.

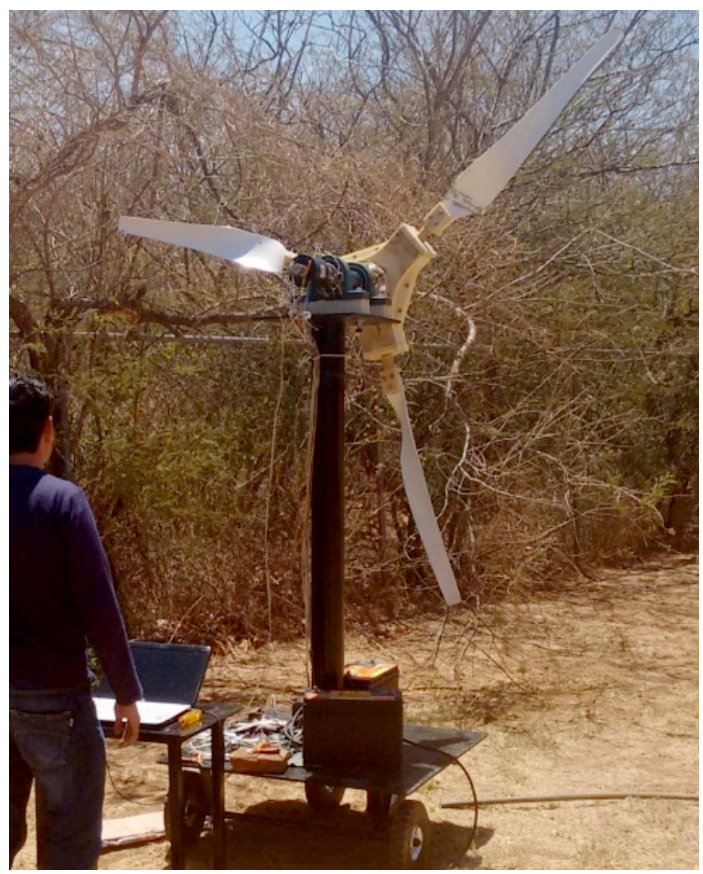

Figura 7. Operación del banco en condiciones no controladas. caso particular de las palas empleadas este ángulo fue de $32^{\circ}$ del plano frontal del cubo. A partir de esta referencia denominado como "ángulo cero", se realizó una variación de la velocidad del viento en el túnel.

En la Figura 8 se muestran las curvas de velocidad del rotor en revoluciones por minuto (RPM) como producto de las variaciones de viento. La velocidad del viento útil para que el rotor comience a operar fue de $3,5 \mathrm{~m} / \mathrm{s}$. El rotor presentó un comportamiento con menor variación en estado estable (90 RPM) para una velocidad de 7,42 m/s, con un tiempo de respuesta del rotor menor a $40 \mathrm{~s}$, lo cual define un punto de operación del sistema para un "ángulo cero".

\section{Caracterización del pitch}

En la prueba de cambio del ángulo de ataque de las palas se operó el túnel de viento a una velocidad promedio de $6,1 \mathrm{~m} / \mathrm{s}$. La respuesta en la velocidad del rotor está en función de la variación del ángulo de pitch (Figura 9). Inicialmente el sistema es operado en "ángulo cero", una vez alcanzado un valor constante, se realizan incrementos en el ángulo. El rotor sufre un freno aerodinámico que hace que la velocidad del giro disminuya hasta el punto de que el sentido del giro del rotor cambia. La velocidad se reduce a cero conforme las palas asumen una posición de bandera.

\section{Implementación de un controlador PI}

A manera de verificar la funcionalidad integral del sistema en la evaluación de esquemas de control por cambio del ángulo de paso, se realizó la implementación de un controlador ProporcionalIntegral (PI) y uno difuso. Se realizó un esquema de simulación en Matlab para establecer los parámetros

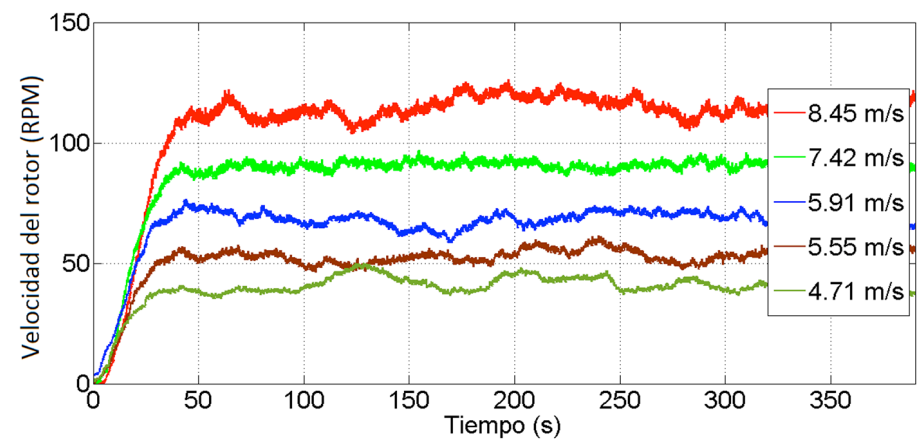

Figura 8. Curvas de velocidad del rotor a variaciones de viento. 


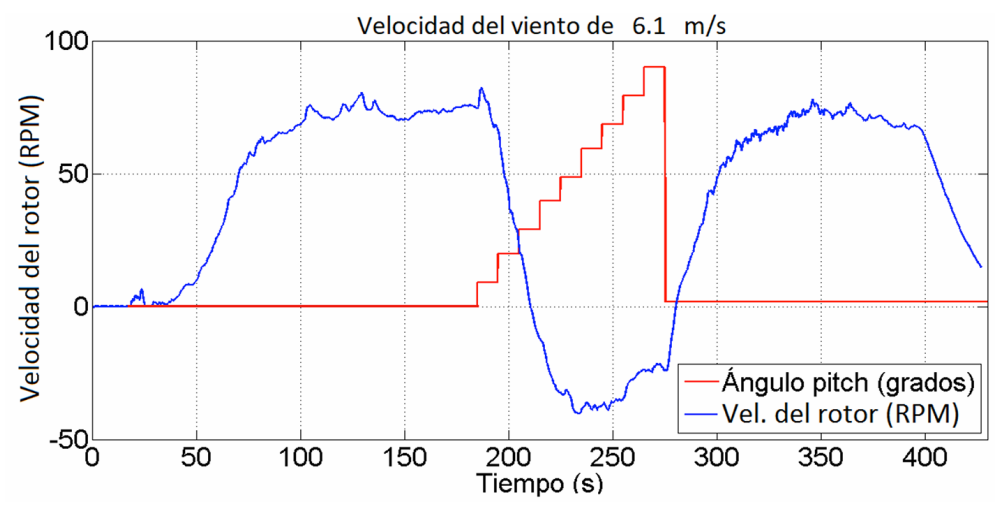

Figura 9. Curvas de velocidad del rotor a variaciones del ángulo de pitch.

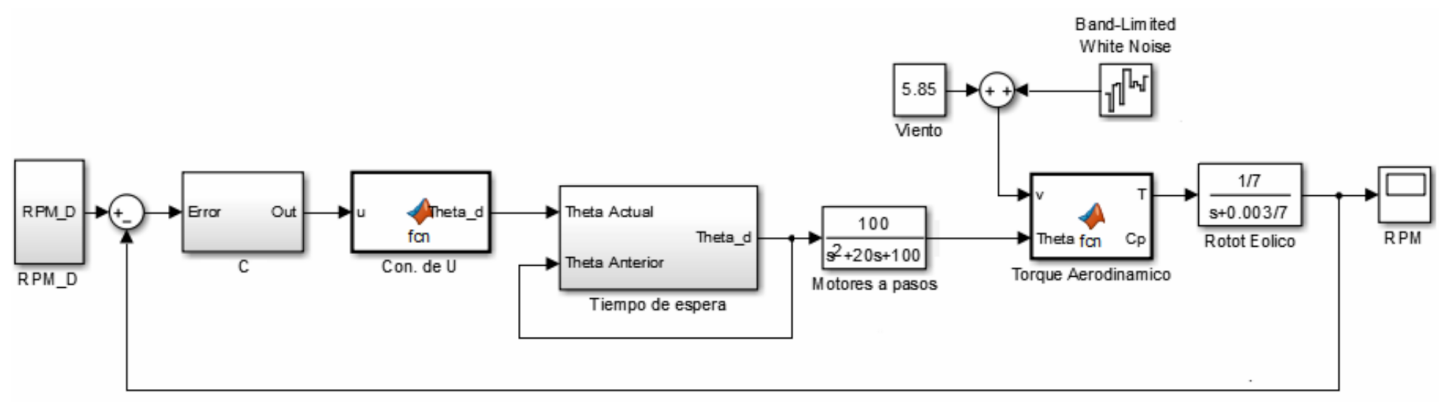

Figura 10. Esquema general de simulación de controladores en Matlab.

iniciales para una sintonización experimental de los controladores (Figura 10). El rotor es modelado como un sistema de primer orden, mientras los motores son caracterizados utilizando un filtro de segundo orden.

Se obtuvieron gráficos experimentales de la respuesta escalón a 60, 80 y 40 RPM (Figura 11), para un controlador PI con ganancias $\mathrm{Kp}=1,5$ y Ki $=0,09$, bajo un escenario con una velocidad de viento promedio de $5,85 \mathrm{~m} / \mathrm{s}$ y un tiempo de muestreo del controlador de $2,5 \mathrm{~s}$.

Con variaciones de escalón de 20 RPM el tiempo de respuesta fue de aproximadamente $10 \mathrm{~s}$, con un error en estado estable de \pm 5 RPM y sobrepasos de la misma escala (Figura 11a). El sobresfuerzo en la señal de control se ve reflejado en las variaciones transitorias en el ángulo del pitch $\theta$ (Figura 11b).

Para el caso del controlador difuso, se planteó el uso de un sistema de inferencia Sugeno con dos variables de entrada (error y velocidad del viento), de cuatro y tres valores lingüísticos respectivamente, y con funciones de membresía sigmoide y gaussiana. La variable de salida (ángulo de paso) se dividió en nueve valores lingüísticos utilizando un sistema Sugeno de orden cero, de modo tal que la función para cada variable estuviera definida como una constante $\beta_{i}$. Los resultados de la acción del controlador difuso a una entrada escalón variable se muestran en la Figura 12. Como se observa el tiempo de respuesta es cercano a $5 \mathrm{~s}$, mientras existe un error en estado estable perdurable, y un mayor amortiguamiento.

Los resultados experimentales muestran un mejor desempeño del controlador PI respecto al error (Figuras 11a y 12a), mientras el sobresfuerzo en la señal de control resulta ser similar entre ambos controladores (Figuras 11 b y 12b). En este punto cabe señalar que en aerogeneradores de mayor tamaño, el ángulo de paso cambia más lentamente debido al tamaño de las palas, lo cual sugiere una menor acción integral para el controlador PI. 


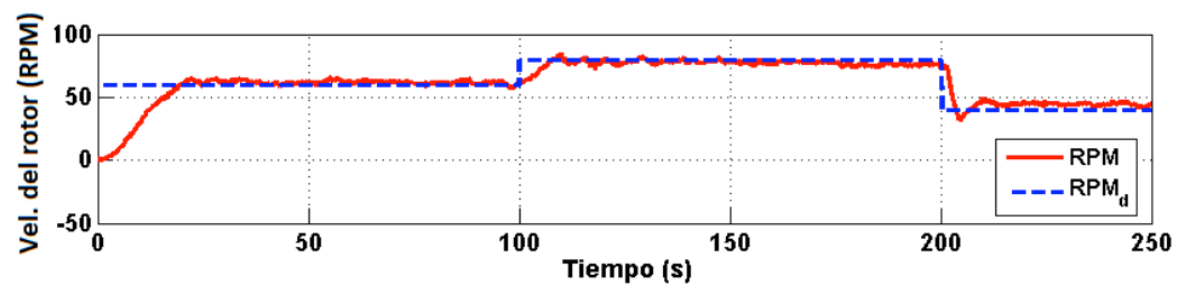

a)

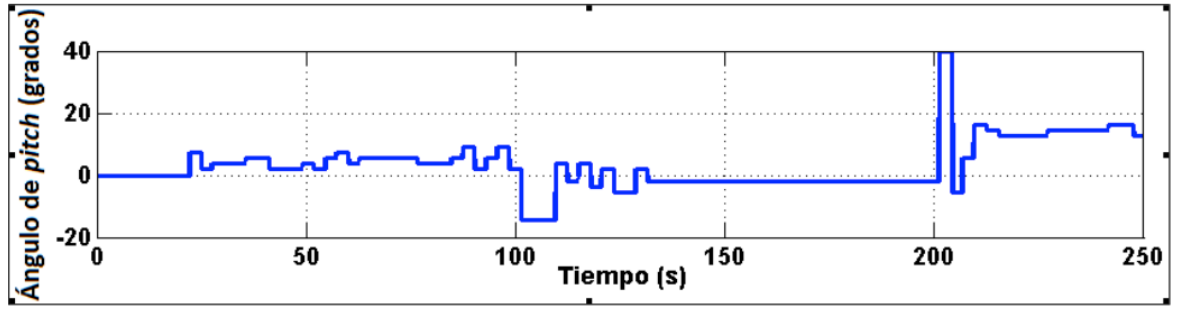

b)

Figura 11. Resultados experimentales de la respuesta escalón de un controlador PI.

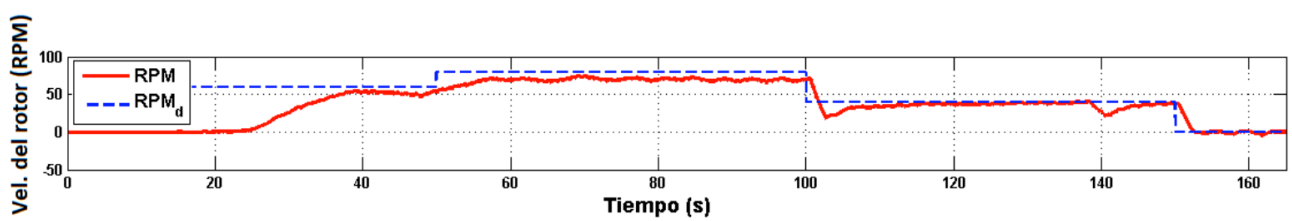

a)

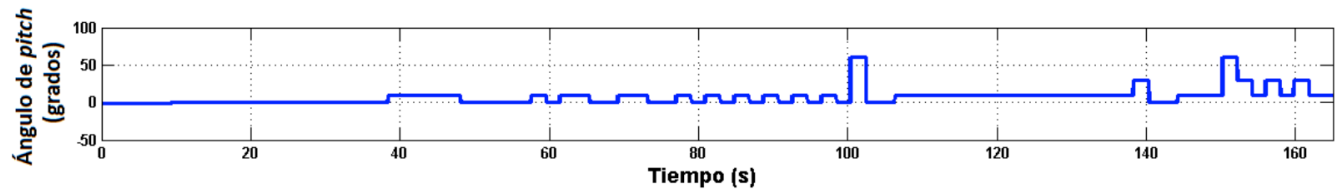

b)

Figura 12. Resultados experimentales de la respuesta escalón de un controlador difuso.

\section{CONCLUSIONES}

En este trabajo se propone el diseño, construcción y caracterización de un banco experimental de pruebas para esquemas de control por cambio del ángulo de paso en aerogeneradores. Dentro de los subsistemas del banco no se considera el eléctrico, por lo que la evaluación de este se centra en la medición de la velocidad en el rotor sin relacionar esta con la energía eléctrica producida.

Bajo la metodología de diseño seleccionada y cuya aplicación es descrita a lo largo de este artículo, el sistema muestra factibilidad y versatilidad en su construcción. De esta forma, el banco también podría ser usado en pruebas de perfiles de palas, a diferencia de bancos de pruebas como los empleados en $[9,10,11]$ que se limitan a simular el torque aerodinámico a través de un motor.

La prestación de la variación del ángulo de ataque en tiempo real para cada pala, contrasta con sistemas similares que requieren de un ajuste mecánico del ángulo previo a las pruebas, por ejemplo [15, 12]; la construcción de diversos hub's como en [14] o no consideran el movimiento individual de las palas como en [8]. 
En pruebas al exterior el rotor del banco mostro movilidad con velocidades de viento menores a $4,5 \mathrm{~m} / \mathrm{s}$, valor típico en aerogeneradores interconectados a la red.

La evaluación del banco en un túnel de viento permitió estimar un punto de operación alrededor de $\operatorname{los} 7,42$ m/s y 90 RPM para un ángulo óptimo. Este valor es comparable con el de microturbinas comerciales de dimensiones similares cuya potencia nominal es alcanzada con una velocidad de viento de $10-13 \mathrm{~m} / \mathrm{s}$.

Por su parte, la variación del ángulo de pitch llevó a estimar el impacto de la regulación del cambio del ángulo de paso en la velocidad y dirección del rotor, lo cual valida el uso del sistema como un banco de prueba experimental. En este sentido, a diferencia de los sistemas comerciales no modificables o caja negra el subsistema de control-monitoreo planteado facilita en gran medida el incremento de prestaciones del software de monitoreo y la implementación de los respectivos algoritmos de control. No obstante, una tarea pendiente es la sustitución de las tecnologías comerciales empleadas por hardware y software libre.

Finalmente, la caracterización de la operación del rotor y del cambio del ángulo de paso, y la implementación de dos tipos diferentes de controladores, sustentan la factibilidad de tareas como la identificación del sistema y el diseño e implementación de controladores basados en modelos experimentales, haciendo posible la validación de una gran variedad de esquemas de control por cambio del ángulo, abordados hasta ahora solo a nivel de simulación.

\section{AGRADECIMIENTOS}

Este trabajo se realizó en el marco del Proyecto CEMIE-Eólico P08 con parte de los recursos financieros del Fondo SENER-CONACYT de Sustentabilidad-Energética.

\section{REFERENCIAS}

[1] O. Anaya-Lara, N. Jenkins, J.B. Ekanayake, P. Cartwright and M. Hughes. "Wind Energy Generation: Modelling and Control". Jhon Wiley \& Sons. ISBN: 978-0-470-71433-1. 2009.
[2] H. Jafarnejadsani, J. Pieper and J. Ehlers. "Adaptive Control of a Variable-Speed Variable-Pitch Wind Turbine Using RadialBasis Function Neural Network". IEEE Transactions on Control Systems Technology. Vol. $21 \mathrm{~N}^{\mathrm{o}}$ 6, pp. 2264-2272. Print ISSN: 1063-6536. Electronic ISSN: 1558-0865. DOI: 10.1109/TCST.2012.2237518.

[3] L. Xianzheng, W. Xingcheng, Z. Kai and L. Jinyuan. "Design of variable pitch-control system based on two-degree-of-freedom internal model control". International Conference on Information Science, Electronics and Electrical Engineering, pp. 1348-1352. Sapporo, Japan. April, 2014. DOI: 10.1109/InfoSEEE.2014.6947893.

[4] K. Qi. "Research on variable pitch wind turbine control system". 2014 IEEE PES Asia-Pacific Power and Energy Engineering Conference (APPEEC), pp. 1-4. Hong Kong, China. December, 2014. DOI: 10.1109/ APPEEC.2014.7066117.

[5] H. Kumar, A. Gupta, R. Pachauri and Y. Chauhan. "PI/FL based blade pitch angle control for wind turbine used in wind energy conversion system". International Conference on Recent Developments in Control, Automation and Power Engineering (RDCAPE), pp. 15-20. Noida, India. October, 2015. DOI: 10.1109/RDCAPE.2015.7281362.

[6] D. Bansal and K. Pandey. "Blade pitch angle and tip speed ratio control schemes for constant power generation of WECS". IEEE 1st International Conference on Power Electronics, Intelligent Control and Energy Systems (ICPEICES), pp. 1-6. Delhi, India. July, 2016. DOI: 10.1109/ ICPEICES.2016.7853498.

[7] Z. Zhichao and W. Chengshan. "Output power curtailment control of variable-speed variable-pitch wind turbine generators". IEEE PES Asia-Pacific Power and Energy Engineering Conference (APPEEC), pp. 1-7. Hong Kong, China. December, 2014. DOI: 10.1109/APPEEC.2014.7065980.

[8] C.M. Sung and M.C. Han. "Design and Performance Evaluation of Hinge Type Pitch Control System in Small-Size Wind Turbine". Eng. and Manuf.-Green Tech. Vol. $3 \mathrm{~N}^{\circ} 4$, pp. 335-341. 2016. DOI: $10.1007 /$ s40684-016-0042-2. 
[9] C.W. Lim. "A demonstration on the similarity of pitch response between MW wind turbine and small-scale simulator". Renewable Energy. 2018. DOI: 10.1016/j. renene.2018.06.008.

[10] M. Yin, W. Li, C.Y. Chung, Z. Chen and Y. Zou. "Inertia compensation scheme of WTS considering time delay for emulating largeinertia turbines". IET Renewable Power Generation. Vol. 11 N$^{\circ}$ 4, pp. 529-538. 2017. DOI: 10.1049/iet-rpg.2016.0782.

[11] X. Tang and M. Yin. "Active Power Control of Wind Turbine Generators via Coordinated Rotor Speed and Pitch Angle Regulation". IEEE Transactions on Sustainable Energy. Vol. $10 \mathrm{~N}^{\mathrm{o}} 2$, pp. 822-832. 2019. DOI: 10.1109/TSTE.2018.2848923.

[12] P.A. Costa, J.W. Carneiro, R.J. Pontes, M.E. Vieira, D. Albiero, C.F. de Andrade and F.O.M. Carneiro. "The Effects of Blade Pitch Angle on the Performance of Small-scale Wind Turbine in Urban Environments". Energy. Vol. 148, pp. 169-178. 2018. DOI: 10.1016/j.energy.2018.01.096.

[13] N. Hara, S. Tsujimoto, Y. Nihei, K. Lijima and K. Konishi. "Experimental validation of model-based blade pitch controller design for floating wind turbines: system identification approach". Wind Energy. Vol. $20 \mathrm{~N}^{\mathrm{o}} 7$, pp. 1187-1206. 2017. ISSN: 1099-1824. DOI: 10.1002/we.2089.

[14] A. Mills, M. Benedict and I. Chopra. "Investigation of the Effect of Blade Kinematics and Reynolds Number on the Aerodynamic Performance of a SmallScale Vertical Axis Wind Turbine with Dynamic Blade Pitching". 54th AIAA Aerospace Sciences Meeting, AIAA
SciTech Forum, pp. 1-11. San Diego, California, USA. January, 2016. DOI: 10.2514/6.2016-0137.

[15] A. Raj, R. Gurav, J. Sankpal, D. Chavan and P. Karandikar. "Study of output parameters of horizontal axis wind turbines using experimental test setup". IEEE 1st International Conference on Power Electronics, Intelligent Control and Energy Systems (ICPEICES), pp. 1-6. Delhi, India. July, 2016. DOI: 10.1109/ ICPEICES.2016.7853120.

[16] M. Moness, M. Osama and A.M. Moustafa. "A Real-time Heterogeneous Emulator of a Highfidelity Utility-scale Variablespeed Variablepitch Wind Turbine". IEEE Transactions on Industrial Informatics. Vol. 14 $\mathrm{N}^{\circ}$ 2, pp. 437-447. 2018. DOI: 10.1109/ TII.2017.2723960.

[17] Y. Gu, Y. Lin, Q. Xu, H. Liu and W. Li. "Blade-pitch system for tidal current turbines with reduced variation pitch control strategy based on tidal current velocity preview". Renewable Energy. Vol. 115, pp. 149-158. 2018. ISSN: 0960-1481. DOI: $10.1016 / j$. renene.2017.07.034.

[18] G. Pahl, W. Beitz, J. Feldhusen and K.H. Grote. "Engineering Design: A Systematic Approach". Springer-Verlag London. ISBN: 978-1-84628-319-2. 2007.

[19] G. Toledo, R. Rosales, M. Hernández and J. Arellano. "Usability test of a SCADA system for monitoring and operating an experimental wind rotor". International Conference on Computing Systems and Telematics (ICCSAT), pp. 1-6. Xalapa, México. October, 2015. DOI: 10.1109/ ICCSAT.2015.7362932. 